Brazilian Journal

of Chemical

ISSN 0104-6632

Printed in Brazil

Engineering

www.scielo.br/bjce

Vol. 35, No. 02, pp. 313 - 326, April - June, 2018

dx.doi.org/10.1590/0104-6632.20180352s20160321

\title{
PVT EXPERIMENTAL AND MODELLING STUDY OF SOME SHALE RESERVOIR FLUIDS FROM ARGENTINA $^{\#}$
}

\author{
Martin Cismondi1 1, , Natalia G. Tassin ${ }^{1}$, Carlos Canel ${ }^{2}$, Francisco \\ Rabasedas $^{2}$ and Carlos Gilardone ${ }^{2}$
}

\begin{abstract}
${ }^{1}$ Instituto de Investigación y Desarrollo en Ingeniería de Procesos y Química Aplicada (IPQA), Universidad Nacional de Córdoba - CONICET. Córdoba, Argentina.

${ }^{2}$ Field Development Consultants (FDC de Argentina SRL). Buenos Aires, Argentina.
\end{abstract}

(Submitted: May 19, 2016; Revised: January 9, 2017; Accepted: January 19, 2017)

\begin{abstract}
Shale reservoir fluids have been receiving much attention, especially during the last decade, due to the important reserves confirmed in many places around the world and the recent production growth in the United States, Argentina, and probably other countries to follow. In some fields of the Vaca Muerta formation in Argentina, the fluids can be classified as gas condensate or near-critical in some cases, presenting retrograde condensation of up to $30 \%$ in volume. This work presents compositional and PVT data of two gas condensate fluids, together with a new methodology for assigning molecular weights and densities to the different carbon number fractions when measured values are available for the whole fluid or liquid phase and only a weight fraction is collected through chromatography for each single cut. A thermodynamic modelling study of these fluids, and also a third one classified as volatile oil, is based on both the PR (Peng and Robinson, 1976) and RKPR (Redlich Kwong Peng Robinson, apud Cismondi and Mollerup, 2005) equations of state, together with different ways of characterizing the heavy fractions. The focus is on phase envelopes, but knowing only the saturation point at the reservoir temperature, and also on retrograde behavior.
\end{abstract}

Keywords: Gas Condensate, RKPR EoS, Phase Envelope, Retrograde Condensation, Characterization.

\section{INTRODUCTION}

Since 2005, and in the context of an important decline in the reserves of conventional hydrocarbon resources, Argentina has been facing the challenge of maintaining the production level of gas, the main component of its energy matrix. The difference between the demand and production curves should be compensated by fluids from new non-conventional reservoirs, mainly Shale Gas.

In many of the recently discovered fields, the produced fluids could be classified as near-critical, with some cases presenting retrograde condensation of around $30 \%$ in volume at the reservoir temperature.

* Corresponding author: martin.cismondi@gmail.com

\# This is an extended version of the work presented at the VIII Brazilian Congress of Applied Thermodynamics - CBTermo 2015, Aracaju, Brazil. 
Material balances and numerical simulations, which are used for predicting the fluids production behavior and proposing development strategies, require an adequate characterization of the reservoir fluids and a good fitting of the laboratory data, in order to adequately reproduce field and well behaviors.

As far as we know, and despite the relevance of the Vaca Muerta formation, no articles have been published so far containing PVT or compositional data on the produced fluids. A Scopus search for the words "Vaca Muerta" by the end of year 2016 returned 24 publications, starting from 2003, all of them related to geological studies of the formation. A second search with the words "Shale" + "Argentina" returned 10 publications, again with focus mainly on geological aspects, and including also two articles published in the Journal of Petroleum Technology, on trends and perspectives about the production of shale gás and shale oil worlwide (Beckwith, 2011). But no specific data on the fluids could be found.

This work presents compositional and PVT data obtained at the ITBA-FDC laboratory for two gas condensate fluids from different fields corresponding to the Vaca Muerta formation in Argentina. The experimental methods are summarized in the next section. Then, a new methodology for assigning molecular weights and densities to the different carbon number fractions is described and implemented to the two fluids and also a third one from the FDC data base, which was classified as volatile oil. Finally, another section is dedicated to a thermodynamic modeling study of the fluids, based on two different cubic equations of state: the PR EoS (Peng and Robinson, 1976) and RKPR EoS (Redlich Kwong Peng Robinson, apud Cismondi and Mollerup, 2005), and exploring the effects of different factors on the equation-of-state description of the fluids, including different ways of representing the heavy residual fraction.

\section{EXPERIMENTAL METHODS AND EQUIPMENT}

The compositional analysis and PVT tests performed at the ITBA-FDC laboratory followed essentially the procedures described in the books by Danesh (1998) and Pedersen and Christensen (2006), for the case of surface separator samples. Chromatographic analyses were carried out according to the norms ASTM D-1945/03, GPA 2286 and ISO 6976/95 for gases, and EPA 8015 and ASTM D-2789 for liquid chromatography. The main equipment involved, for the fluids reported in this work, were the following: (a) PVT cell "DBR-Jefri", serial 0150-100-200, maximum pressure: $10000 \mathrm{psi}$, maximum temperature: $200{ }^{\circ} \mathrm{C}$; (b) chromatograph HP6890 G1530A with a capillary column HP-PONA Methyl Siloxane HP 19091S-001; (c) chromatograph HP6890 G1530A with a capillary column HP-PONA Methyl Siloxane HP 19091S-001 and a packed column DC200-PO-TM - HP 19006 80005. With both chromatographs the carrier was hydrogen, with a FID front detector and TCD back detector.

The reservoir fluid tested in the PVT cell is the result of the recombination of a gas sample and a liquid sample taken from the separator at the surface of the well, according to the gas-oil ratio (GOR), which is also obtained from the flows measured at the surface separator. Since the liquid sample is taken at the separator pressure, it also needs to be flashed before analyzing its composition. Then, the steps leading to the analytical determination of the reservoir fluid composition are the following:

- Determination of the separator gas composition by chromatography.

- Determination of the separator liquid composition through the following procedure:

- A flash separation of the liquid sample is performed at atmospheric pressure and room temperature, obtaining the so called flash gas and flash liquid.

- The volumes of the two recovered phases are measured and the gas-liquid ratio is obtained.

- The compositions of the flash gas and liquid phases are determined by chomatography.

- The average molecular weight for the flash liquid is determined by the melting point depression method, using benzene as solvent.

- The flash liquid density is measured using a pycnometer and a high precision weighing scale.

- The separator liquid composition is determined from a recombination of the flash gas and liquid compositions, in a similar way to what is described, for example, in section 2.2 of the book by Pedersen and Christensen (2006).

- Finally, the reservoir fluid composition is determined from the recombination of the separator gas and liquid phases.

Tables 1 and 2 present the compositions of two different gas condensate fluids analyzed at the ITBAFDC laboratory as described above. Note that only mass fractions were obtained from chromatography for C6 and heavier fractions. Molecular weights, mole fractions and densities at standard conditions were 
assigned according to the methodology described in the next section. The five pseudo-components at the end of each Table correspond to the decomposition of the residual $\mathrm{C} 20+$ fraction. More details are given in the next section and specifically in Table 6 .

Table 1: Composition for the Gas Condensate fluid identified as $\mathrm{GC} 1$ in this work

\begin{tabular}{|c|c|c|c|}
\hline Component & $\begin{array}{c}\text { Molar } \\
\text { Fraction }\end{array}$ & $\begin{array}{c}\text { Molecular } \\
\text { Weight }\end{array}$ & $\begin{array}{r}\text { Density } \\
\left(\mathrm{g} / \mathrm{cm}^{3}\right)\end{array}$ \\
\hline Nitrogen & 0.01744 & 28.01 & \\
\hline Carbon Dioxide & 0.00042 & 44.01 & \\
\hline Methane & 0.75731 & 16.04 & \\
\hline Ethane & 0.08026 & 30.07 & \\
\hline Propane & 0.03625 & 44.09 & \\
\hline iso-Butane & 0.00652 & 58.12 & \\
\hline n-Butane & 0.01372 & 58.12 & \\
\hline iso-Pentane & 0.00516 & 72.15 & \\
\hline n-Pentane & 0.00596 & 72.15 & \\
\hline C6 & 0.00877 & 84.00 & 0.685 \\
\hline $\mathrm{C} 7$ & 0.01263 & 96.63 & 0.710 \\
\hline $\mathrm{C} 8$ & 0.01255 & 109.27 & 0.732 \\
\hline $\mathrm{C} 9$ & 0.00739 & 121.90 & 0.753 \\
\hline $\mathrm{C} 10$ & 0.00592 & 134.53 & 0.771 \\
\hline $\mathrm{C} 11$ & 0.00426 & 147.16 & 0.788 \\
\hline $\mathrm{C} 12$ & 0.00361 & 159.80 & 0.803 \\
\hline $\mathrm{C} 13$ & 0.00321 & 172.43 & 0.817 \\
\hline $\mathrm{C} 14$ & 0.00275 & 185.06 & 0.829 \\
\hline $\mathrm{C} 15$ & 0.00247 & 197.69 & 0.840 \\
\hline $\mathrm{C} 16$ & 0.00198 & 210.33 & 0.851 \\
\hline $\mathrm{C} 17$ & 0.00184 & 222.96 & 0.860 \\
\hline $\mathrm{C} 18$ & 0.00139 & 235.59 & 0.868 \\
\hline $\mathrm{C} 19$ & 0.00126 & 248.22 & 0.876 \\
\hline $\mathrm{C} 20+$ & 0.00694 & 328.44 & 0.907 \\
\hline Pseudo-component 1 & 0.00200 & 266.64 & 0.885 \\
\hline Pseudo-component 2 & 0.00142 & 291.90 & 0.896 \\
\hline Pseudo-component 3 & 0.00141 & 322.60 & 0.907 \\
\hline Pseudo-component 4 & 0.00121 & 370.31 & 0.920 \\
\hline Pseudo-component 5 & 0.00090 & 476.99 & 0.935 \\
\hline
\end{tabular}

In order to study the PVT behavior of each reservoir fluid, a Constant Mass Experiment (CME) is performed. This experiment is also referred to as a Constant Composition Expansion (CCE) or simply as a Pressure-Volume (PV) test. Irrespective of the fluid type, it is common practice to carry out a CME test at the reservoir temperature. This study consists of successive expansions of the PVT cell to reproduce the evolution of the fluid at the reservoir temperature. In this evolution, the dew point pressure and the amount
Table 2: Composition for the Gas Condensate fluid identified as GC2 in this work

\begin{tabular}{|c|c|c|c|}
\hline Component & $\begin{array}{c}\text { Molar } \\
\text { Fraction }\end{array}$ & $\begin{array}{c}\text { Molecular } \\
\text { Weight }\end{array}$ & $\begin{array}{r}\text { Density } \\
\left(\mathrm{g} / \mathrm{cm}^{3}\right)\end{array}$ \\
\hline Nitrogen & 0.00387 & 28.01 & \\
\hline Carbon Dioxide & 0.01033 & 44.01 & \\
\hline Methane & 0.62955 & 16.04 & \\
\hline Ethane & 0.12575 & 30.07 & \\
\hline Propane & 0.06768 & 44.09 & \\
\hline iso-Butane & 0.01428 & 58.12 & \\
\hline n-Butane & 0.02658 & 58.12 & \\
\hline iso-Pentane & 0.00955 & 72.15 & \\
\hline n-Pentane & 0.00996 & 72.15 & \\
\hline C6 & 0.01439 & 84.00 & 0.685 \\
\hline $\mathrm{C} 7$ & 0.01707 & 96.69 & 0.709 \\
\hline $\mathrm{C} 8$ & 0.02186 & 109.38 & 0.730 \\
\hline C9 & 0.01205 & 122.06 & 0.750 \\
\hline $\mathrm{C} 10$ & 0.00736 & 134.75 & 0.767 \\
\hline $\mathrm{C} 11$ & 0.00523 & 147.44 & 0.783 \\
\hline $\mathrm{C} 12$ & 0.00403 & 160.13 & 0.797 \\
\hline $\mathrm{C} 13$ & 0.00365 & 172.81 & 0.810 \\
\hline $\mathrm{C} 14$ & 0.00297 & 185.50 & 0.822 \\
\hline $\mathrm{C} 15$ & 0.00252 & 198.19 & 0.833 \\
\hline $\mathrm{C} 16$ & 0.00200 & 210.88 & 0.843 \\
\hline $\mathrm{C} 17$ & 0.00188 & 223.56 & 0.851 \\
\hline $\mathrm{C} 18$ & 0.00138 & 236.25 & 0.859 \\
\hline $\mathrm{C} 19$ & 0.00121 & 248.94 & 0.866 \\
\hline $\mathrm{C} 20+$ & 0.00485 & 313.63 & 0.892 \\
\hline Pseudo-component 1 & 0.00095 & 261.63 & 0.873 \\
\hline Pseudo-component 2 & 0.00138 & 279.97 & 0.881 \\
\hline Pseudo-component 3 & 0.00089 & 305.34 & 0.891 \\
\hline Pseudo-component 4 & 0.00095 & 340.69 & 0.901 \\
\hline Pseudo-component 5 & 0.00068 & 427.20 & 0.917 \\
\hline
\end{tabular}

of retrograde liquid deposited in the cell at pressures below the dew point are determined. The methodology is as follows:

- A physical recombination from the separator samples is charged to a Robinson PVT cell. This double-window cell allows a direct observation of the fluid and measurement of the volume of condensed liquid. The cell is kept at the reservoir temperature and the fluid is pressurized through a hydraulic piston.

- The monophasic condition of the sample is verified. A visual confirmation is enough for gas condensate fluids, while an additional verification of the compressibility through a differential pressure manometer is usual with oils. 
- The total volume is registered at different pressures, measuring also the volume of retrograde liquid accumulated at pressures below the dew point, which is determined visually.

- The curves pressure vs. relative volume, as well as $\%$ of retrograde liquid vs. pressure are determined.

Regarding experimental uncertainties in the PVT tests, it is difficult to estimate a level of accuracy for the different values informed, since several stages are involved and different factors can affect the results. Nevertheless, based on the repeatibility experience accumulated at the ITBA-FDC lab, the assumed compositional errors are given in Table 3 , together with uncertainties for the different measurements associated with the CME test.

Table 3: Experimental uncertainties associated with the mole fractions determined for defined compounds and measurements in the CME test.

\begin{tabular}{ccc}
\hline \multicolumn{2}{c}{ Variable } & Error \\
\hline Mole fractions in & $\mathrm{x}, \mathrm{y}<0.01$ & $+/-10 \%$ \\
liquid or vapor & $0.01<\mathrm{x}, \mathrm{y}<0.03$ & $+/-5 \%$ \\
phase & $0.03<\mathrm{x}, \mathrm{y}$ & $+/-1 \%$ \\
& Volume & $+/-0.01 \%\left(0.01 \mathrm{~cm}^{3}\right)$ \\
CME test & Pressure & $+/-0.1 \mathrm{~atm}$ \\
& Temperature & $+/-0.1{ }^{\circ} \mathrm{C}$ \\
\hline
\end{tabular}

\section{A new methodology for assigning molecular weights and densities}

In some compositional studies of reservoir fluids (see for example Pedersen et al., 1992) average molecular weights are calculated from the chromatographic single-component distributions for the C6-C9 fractions, and measured by freezing point depression for the distillate fractions obtained for higher cuts through True Boiling Point (TBP) analysis.

Nevertheless, in routine PVT studies like the ones reported here, it is more common that only a mass fraction can be assigned to each cut from C6 on, based on measured quantities. Density at standard conditions and molecular weight are measured only for each separator phase, and sometimes also for the $\mathrm{C} 7+$ or $\mathrm{C} 10+$ fractions.

Then, reported values for single carbon number (SCN) fractions are either estimated based on correlations, or taken from Tables of recommended generalized values. This has been a common practice in the industry and a popular and typical source for generalized density and molecular weight values for single carbon number fractions is the work of Katz and Firoozabadi
(1978), whose table is also included in books covering these topics, like the one by Pedersen and Christensen (2006, see Table 2.1 in the book) .

Those values may work quite well in many cases, providing reasonable values for the residual fraction, e.g., $\mathrm{C} 20+$, but may also lead to unreasonable estimations for the residual fractions when the real values of densities or molecular weights for the SCN fractions have important differences with the assumed generalized values. In the latter case, a consistent characterization of the residual fraction (typically $\mathrm{C} 20+$ ) will be clearly hindered, since solving the equations that equal average values with measured density or molecular weight for the whole fluid makes it accumulate the errors in the previous SCN fractions, for which it will have to compensate.

Therefore, in this work we propose a new methodology for the estimation of molecular weights and densities for single carbon cuts from C6 on, based on measured values for the fluid and general assumptions on the distribution or evolution of these properties along SCN fractions in reservoir fluids.

It is a well established fact that the diversity of molecular structures within a given SCN fraction increases with carbon number. For example, most C6 fractions consist mainly of $n$-hexane, plus some cyclopentane and different quantities of a few branched hexanes. On the other hand, as it is illustrated for example in Table 2.4 in the book of Pedersen and Christensen (2006), a C9 fraction already includes typically more than 20 different compounds detected by chromatography, including normal and branched paraffinic, naphthenic and aromatic structures. For fractions around C20 there can be various dozens of different compounds, with very small quantities of each, and it could be very difficult to achieve a complete list for a specific fluid. As a consequence of that, there is normally no risk in assuming typical values for the density and molecular weight of $\mathrm{C} 6$, while the differences can be important for higher cuts. For that reason, we adopt the recommended values from Katz and Firoozabadi only for C6, and assume specific mathematical functionalities in terms of carbon number for the rest, depending on only one parameter for each property, which can be adjusted in order to match the measured value for the whole fluid.

For molecular weights a linear relation with carbon number is normally accepted. In our methodology, following the reasoning above, the corresponding equation for estimating the SCN molecular weights is:

$$
M_{i}=84+C(i-6)
$$


where $i$ is a number identifying a SCN fraction and $C$ is an adjustable constant.

The relation with density is not that simple. Pedersen, for example, recommends a linear relation with the logarithm of the carbon number. Instead, in this work, we use the following equation with $A_{d}$ and $B_{d}$ being adjustable constants:

$\rho_{i}=A_{d} * e^{-i / 10}+B_{d}$

For this relation we have found a better correlation capacity when considering experimental values, including those recommended by Katz and Firoozabadi (1978).

Fixing this equation to the typical recommended value for C6, there is only one degree of freedom left, which can be associated with the $A_{d}$ parameter, since $B_{d}$ becomes

$B_{d}=0.685-A_{d} * e^{-0.6}$

Finally, as it is well established that there exist a high linearity between the logarithm of molar fractions and the carbon number of the corresponding cut (Pedersen et al., 1992), the following equation will be adopted for the distribution of the $\mathrm{C} 20+$ fraction:

$\ln \left(z_{i}\right)=A * i+B$

So, Eqs. (1-4) are our working equations, and the proposed procedure for decomposing the available mass fractions and assigning densities for the different $\mathrm{SCN}$ fractions is as follows:

1) Find the value of $C$ for Eq. (1) that leads to a consistent distribution of fractions, where an extrapolation to the $\mathrm{C} 20+$ range is in agreement with the measured values for the fluid. This requires the following sub-steps:

a. Obtain the value of $z_{6+}$ and products $\left(\mathrm{z}^{*} \mathrm{M}\right) \mathrm{i}$ based on the experimental information available.

b. For a given value of $\mathrm{C}$, calculate the values of the $M_{i}$ 's ( $i=6$ to 19) from Eq. (1), and the corresponding mole fractions as $z_{i}=\left(\mathrm{z}^{*} \mathrm{M}\right)_{\mathrm{i}} / \mathrm{M}_{\mathrm{i}}$.

c. Calculate $z_{20+}=z_{6+}-\sum_{i=6}^{19} z_{i}$

d. Calculate $M_{20+}=(z * M)_{20+} / z_{20+}$

e. Obtain the Best Feasible Extrapolation (Ramello and Cismondi, 2016) based on mole fractions from step $b$, providing the coefficients $\mathrm{A}$ and $\mathrm{B}$ for the distribution (Eq. 4), and find the corresponding $\mathrm{Cmax}$ where the summation reaches $z_{20+^{*}}$ f. Based on the estimated distribution of the $\mathrm{C} 20+$ fraction from the previous point, obtain the corresponding $\mathrm{M}_{20+}^{\text {calc }}=\sum_{i=20}^{C_{\max }} z_{i} * M / z_{20+}$. If it is not equal to the value from step d, estimate a new value for $\mathrm{C}$, e.g., following the secant method, and go back to step b.

2) Find the value of $A_{d}$ for Eq. (2) that leads to recovering the density of the fluid. This requires the following sub-steps:

a. Obtain the experimental value of $V_{6+}$ (see Nomenclature section), depending on the information available. With a report with density values assigned somehow, it is calculated as $V_{6+}=\sum_{i=6}^{19} \frac{z_{i} M_{i}}{\rho_{i}}+\frac{z_{20+} M_{20+}}{\rho_{20+}}$. The following steps aim at a sort of redistribution of the densities (or volumes), considering that arbitrary values, e.g. those from Katz and Firoozabadi (1978), will be replaced by a continuous function like Eq. (2) which, when applied from C6 to Cmax, can consistently recover the density measured for the fluid.

b. For a given value of $A_{d}$, obtain the correspon$\operatorname{ding} B_{d}$ from Eq. (3) and calculate the values of the $\rho_{\mathrm{i}}^{\prime} \mathrm{s}(\mathrm{i}=6$ to $\mathrm{Cmax}$ ) from Eq. (2).

c. Obtain $V_{6+, \text { calc }}=\sum_{i=6}^{C_{\max }} \frac{z_{i} M_{i}}{\rho_{i}}$.

d. If it is not equal to the $V_{6+}$, estimate a new value for $A_{d}$, e.g., following the secant method, and go back to step $b$.

3) Lump the $\mathrm{C} 20+$ distribution into Nps pseudo-components having similar mass fractions or $\mathrm{Z}^{*} \mathrm{M}$ products. In this work $\mathrm{Nps}=5$.

Note that the products $\left(\mathrm{z}^{*} \mathrm{M}\right)_{\mathrm{i}}$ are available based on measurements, from the equation:

$$
(z * M)_{i}=m_{i} / n_{\text {total }} \text { where } n_{\text {total }}=m_{\text {total }} / M_{\text {total }}
$$

and total can refer to $\mathrm{C} 6+, \mathrm{C} 10+$ or the complete fluid, etc., depending on available measurements.

The procedure has been explained for fluids analyzed up to $\mathrm{C} 20+$, but would be equally applicable to cases with $\mathrm{C} 30+$ or $\mathrm{C} 35+$.

Regarding the concept of Best Feasible Extrapolation referred to in step 1d, its detailed explanation is part of a parallel article to be published soon (Ramello and Cismondi, 2017). Nevertheless, it essentially implies that an extrapolation based on Eq. (4) and pre-20 mole fraction values will be applied to the $20+$ range as long as the slope falls in between the two feasibility limits alluded to in the following lines. If the slope is too pronounced, the distribution along 
carbon numbers will go up to infinity without ever reaching $z_{20+}$. In this case, the limiting slope for which the summation of mole fractions reaches $z_{20+}$ at infinity (in practice, a high carbon number) will be adopted. On the other hand, if the slope is too small very low and unrealistic Cmax values can be obtained. In these cases, a slope leading to a Cmax near 60 is adopted.

Table 4 presents the values of the different parameters involved for each of the analyzed gas condensate fluids, and also a volatile oil from the FDC data base (VO in this work), whose composition and different assigned values are given in Table 5. Details on the decomposition of the $\mathrm{C} 20+$ fractions, including carbon numbers covered by each pseudo-compound and distribution of the $\mathrm{z}^{*} \mathrm{M}$ products, are given in Table 6 .

Table 4: Parameters related to the proposed molecular weight and density functions, together with the distribution of the $\mathrm{C} 20+$ fraction, for the three fluids studied in this work.

\begin{tabular}{cccc}
\hline Fluid & GC1 & GC2 & VO \\
\hline $\mathrm{z}_{6+}$ & 0.07696 & 0.10245 & 0.24005 \\
$\mathrm{C}$ (Eq. 1) & 12.6325483 & 12.6875097 & 14.2892066 \\
$\mathrm{~A}$ (Eq. 4) & -0.1696998 & -0.21744749 & -0.15788421 \\
$\mathrm{~B}$ (Eq. 4) & -3.43306907 & -2.61117457 & -2.31599058 \\
$\mathrm{C}_{\max }$ & 58 & 56 & 59 \\
$\mathrm{~A}_{\mathrm{d}}$ (Eq. 2) & -0.47725489 & -0.45407753 & -0.40630662 \\
$\mathrm{~B}_{\mathrm{d}}$ (Eq. 2) & 0.94692303 & 0.93420303 & 0.90798579 \\
$\mathrm{z}_{20+}$ & 0.00694 & 0.00485 & 0.02867 \\
$\mathrm{M}_{20+}$ & 328.44 & 313.63 & 366.43 \\
$\rho_{20+}$ & 0.907 & 0.892 & 0.876 \\
\hline
\end{tabular}

Table 5: Composition for the Volatile Oil identified as VO in this work.

\begin{tabular}{cccc}
\hline Component & $\begin{array}{c}\text { Molar } \\
\text { Fraction }\end{array}$ & $\begin{array}{c}\text { Molecular } \\
\text { Weight }\end{array}$ & $\begin{array}{c}\text { Density } \\
\left(\mathbf{g} / \mathbf{c m}^{3}\right)\end{array}$ \\
\hline Nitrogen & 0.00525 & 28.01 & \\
Carbon Dioxide & 0.00193 & 44.01 & \\
Methane & 0.51688 & 16.04 & \\
Ethane & 0.10377 & 30.07 & \\
Propane & 0.06374 & 44.09 & \\
iso-Butane & 0.01214 & 58.12 & \\
n-Butane & 0.03087 & 58.12 & \\
iso-Pentane & 0.01123 & 72.15 & \\
n-Pentane & 0.01414 & 72.15 & \\
C6 & 0.02090 & 84.00 & 0.685 \\
C7 & 0.03031 & 98.29 & 0.706 \\
C8 & 0.03803 & 112.58 & 0.725 \\
C9 & 0.02621 & 126.87 & 0.743
\end{tabular}

\begin{tabular}{cccc} 
C10 & 0.01964 & 141.16 & 0.759 \\
C11 & 0.01438 & 155.45 & 0.773 \\
C12 & 0.01111 & 169.74 & 0.786 \\
C13 & 0.01073 & 184.02 & 0.797 \\
C14 & 0.00905 & 198.31 & 0.808 \\
C15 & 0.00827 & 212.60 & 0.817 \\
C16 & 0.00661 & 226.89 & 0.826 \\
C17 & 0.00595 & 241.18 & 0.834 \\
C18 & 0.00546 & 255.47 & 0.841 \\
C19 & 0.00473 & 269.76 & 0.847 \\
C20+ & 0.02867 & 366.43 & 0.876 \\
Pseudo-component 1 & 0.00778 & 290.63 & 0.855 \\
Pseudo-component 2 & 0.00567 & 319.21 & 0.865 \\
Pseudo-component 3 & 0.00576 & 354.00 & 0.874 \\
Pseudo-component 4 & 0.00519 & 408.19 & 0.885 \\
Pseudo-component 5 & 0.00426 & 533.63 & 0.898 \\
\hline
\end{tabular}

Table 6: Decomposition of the $\mathrm{C} 20+$ fraction from each fluid into 5 pseudo-components.

\begin{tabular}{ccccccc|}
\hline $\begin{array}{c}\text { Pseudo- } \\
\text { component }\end{array}$ & $\begin{array}{c}\text { GN } \\
\text { range }\end{array}$ & $\mathbf{z}^{* \mathbf{M}}$ & $\begin{array}{c}\text { CN } \\
\text { range }\end{array}$ & $\mathbf{z}^{* \mathbf{M}}$ & $\begin{array}{c}\text { CN } \\
\text { range }\end{array}$ & $\mathbf{z}^{* \mathbf{M}}$ \\
\hline 1 & $20-21$ & 0.533 & 20 & 0.249 & $20-21$ & 2.261 \\
2 & $22-23$ & 0.414 & $21-22$ & 0.386 & $22-23$ & 1.810 \\
3 & $24-26$ & 0.455 & $23-24$ & 0.272 & $24-26$ & 2.039 \\
4 & $27-31$ & 0.448 & $25-28$ & 0.324 & $27-31$ & 2.119 \\
5 & $32-58$ & 0.429 & $29-56$ & 0.290 & $32-59$ & 2.273 \\
\hline
\end{tabular}

Figs. 1 and 2 show the molecular weight and density functions obtained, in comparison to the generalized values from Katz and Firoozabadi (1978) and also some ranges of expected values. For fractions from C6 to $\mathrm{C} 9$ these ranges are given by the values corresponding to the single components that can be found, according to Table 2.4 in the book of Pedersen and Christensen (2006), while the ranges for C19 covers the values found in more than 30 reservoir fluids taken from the works of Pedersen et al. (1992), Jaubert et al. (2002) and also Chapter 3 in the book of Pedersen and Christensen (2006). In comparison to those ranges and generalized values, the trends resulting from our proposed methodology appear to be reasonable. The two gas condensates GC1 and GC2 show nearly the same line for molecular weights and similar curves for densities. The volatile oil presents the higher molecular weights and lower densities, which could be an indication of a more paraffinic fluid than the others. 


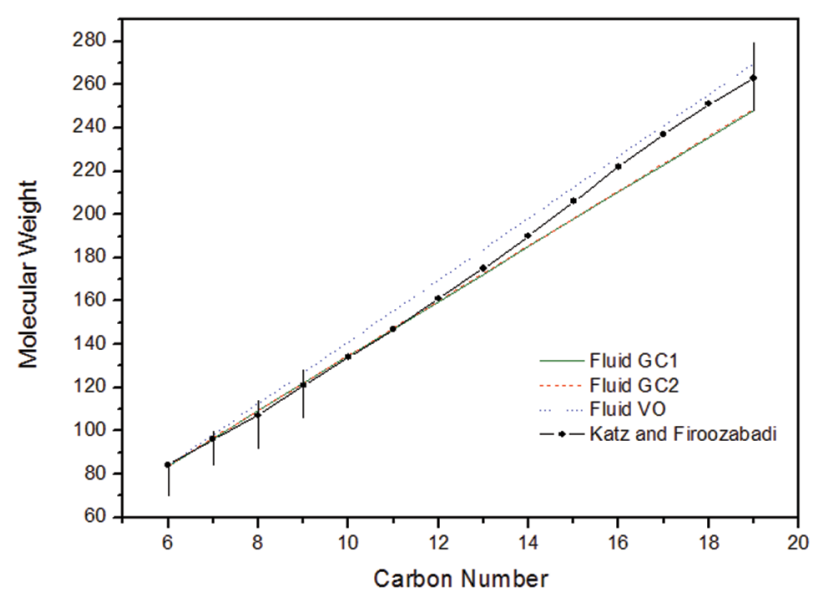

Figure 1: Molecular weight values assigned to the reservoir fluids according to the proposed methodology. Generalized values recommended by Katz and Firoozabadi (1978) are included for comparison. Vertical bars indicate ranges of expected values (see text).

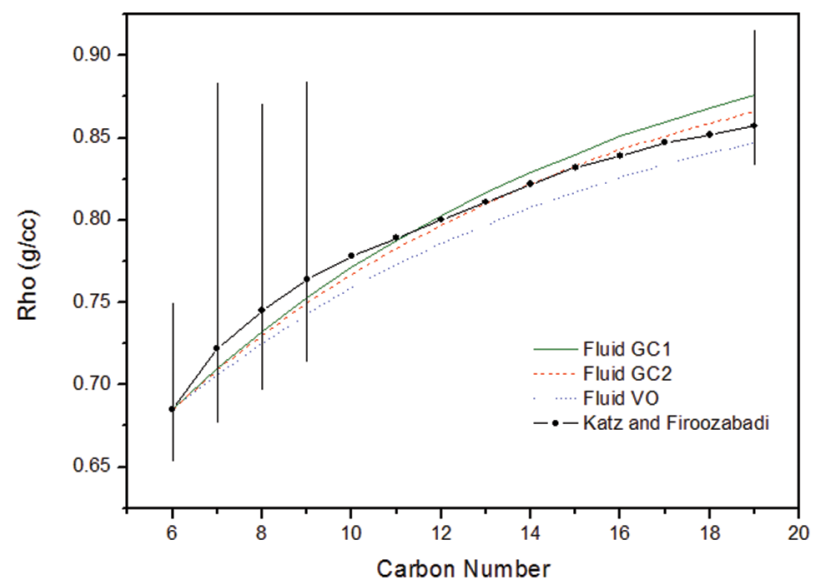

Figure 2: Density values assigned to the reservoir fluids according to the proposed methodology. Generalized values recommended by Katz and Firoozabadi (1978) are included for comparison. Vertical bars indicate ranges of expected values (see text).

\section{THERMODYNAMIC MODELLING}

In this work we do not have the goal of achieving the best match or description of some behaviour for the specific fluids studied. Instead, we rather want to use these cases in order to analyze how different aspects or parameters in the equation-of-state modelling of PVT properties may affect the results. Then, this study is meant to provide some insight for future modelling works concerned with these types of reservoir fluids, and probably help in guiding the definition of new strategies for modelling algorithms.
There are mainly three factors affecting the calculation of PVT properties, once an equation of state has been chosen to model a given reservoir fluid of known composition, like the ones considered in this work:

I) How to represent the residual heavy fraction, in these cases $\mathrm{C} 20+$. Just as one pseudo-component? Decompose it? Into how many pseudo-components and how?

II) Critical temperatures and pressures $\left(\mathrm{T}_{\mathrm{C}}, \mathrm{P}_{\mathrm{C}}\right)$, and also acentric factors, assigned to each pseudo-component from C6 on, typically as functions of density and molecular weight of each given fraction, and then eventually adjusted through some matching procedure.

III) Interaction parameters: typically the kij's (attractive ones) and alternatively lij's (repulsive, not used in the common practice with classic models like PR).

In this study we defined and implemented three different approaches in order to roughly see the effects of choices regarding factors I and II:

Approach A: Fractions C6 to C19 are represented by n-alkanes with the same carbon numbers. The $\mathrm{C} 20+$ fraction is represented by another single n-alkane, the one with closest molecular weight.

Approach B: $\mathrm{T}_{\mathrm{C}}$ and $\mathrm{P}_{\mathrm{C}}$ for the pseudo-components representing fractions from $\mathrm{C} 6$ on are estimated by Pedersen correlations based on MW and density. The same for the acentric factor, using the correlation designed for PR. The $\mathrm{C} 20+$ is treated the same way, as if it were a single carbon number.

Approach C: Idem to Approach B, except that the $\mathrm{C} 20+$ fraction is split into five pseudo-components with similar mass fractions. Details for the fluids studied here are provided in Table 6.

Note that Approach A, the simplest one, may be the least realistic among the three studied approaches. But it was considered in order to see -based on comparisons with Approach B- the effect of factor II on calculations. Similarly, comparison between approaches B and $\mathrm{C}$ will show the effects of decomposing the $\mathrm{C} 20+$ fraction (factor I).

In relation to factor III, we used approaches A to $\mathrm{C}$ in two different ways: first a purely predictive mode, with default alkane-alkane interaction parameters taken from a previous work (Cismondi Duarte et al., 2015, adapted also to SCN cuts in approaches B and C as explained below). And then a matching mode, where a factor $\mathrm{f}$ affects all default interaction parameters (both $k_{i j}$ and $l_{i j}$ for RKPR, $k_{i j}$ for PR), and its value is defined such that the experimental saturation pressure at the reservoir temperature is matched. 
Besides using estimated critical constants and acentric factors for any SCN fraction or pseudo-component in approaches $\mathrm{B}$ and $\mathrm{C}$, their default parameters in the predictive mode were assigned according to the following rules:

- For the $\delta 1$ parameter (RKPR EoS) the following correlation defined by Cismondi Duarte et al. (2015) for n-alkanes is adapted here for pseudo-components based on their molecular weights:

$\delta_{1}=0.91+0.33 * \mathrm{CN}^{\mathrm{Eff} *} e^{\left(-\frac{\mathrm{CN}^{\mathrm{Ef}}}{11}\right)} ; \quad \mathrm{CN}^{\mathrm{Eff}}=\frac{M-2}{14}$

- Interaction parameters $k_{i j}$ (with methane and propane) and $l_{i j}$ (with all lighter defined alkanes) are estimated using the same RKPR correlations proposed for n-alkanes by Cismondi Duarte et al. (2015), dependent on the $a_{c}$ and $\delta_{1}$ parameters of the components involved, respectively.

- All interaction parameters between pseudo-components are zero.

- For the PR EoS all $l_{i j}$ interactions are zero, while $k_{i j}$ 's with the lighter components are estimated based on correlations like the ones used by Cismondi Duarte et al. (2015), but using an effective carbon number, calculated as (M-2)/14, instead of the nominal carbon number.

Table 7 presents the required values of $f$ with the different approaches and two models, for the three fluids considered. Only in two cases (Approaches B and $\mathrm{C}$ with RKPR for fluid GC1) was it not possible to match the saturation pressure with a positive $f$ value. Therefore, $f=0.50$ (giving the lowest error with Approach $\mathrm{B}$ ) and $f=0$ (null interaction parameters for Approach $\mathrm{C}$, in order not to invert the sense of the default values) were applied for making the comparisons.

Figs. 3 to 8 show the predicted phase envelopes for the three fluids, with both the pure predictive and the matching mode. There is a clear trend showing that the extent of predicted immiscibility, and consequently also the predicted saturation pressure, increases from approach A to B and from B to C.

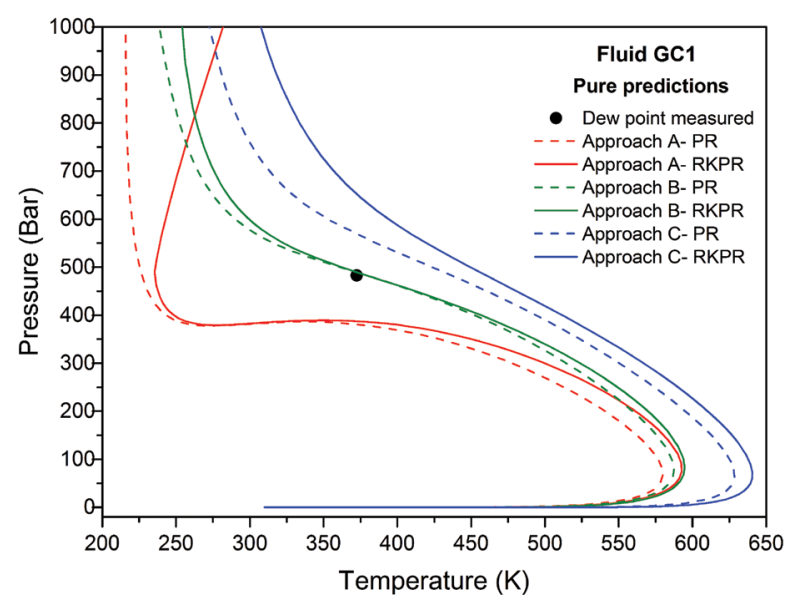

Figure 3: Pure predictions of the phase envelope for fluid GC1, with the PR and RKPR equations of state and the three approaches considered. Default interactions for alkanes assigned to all fractions.

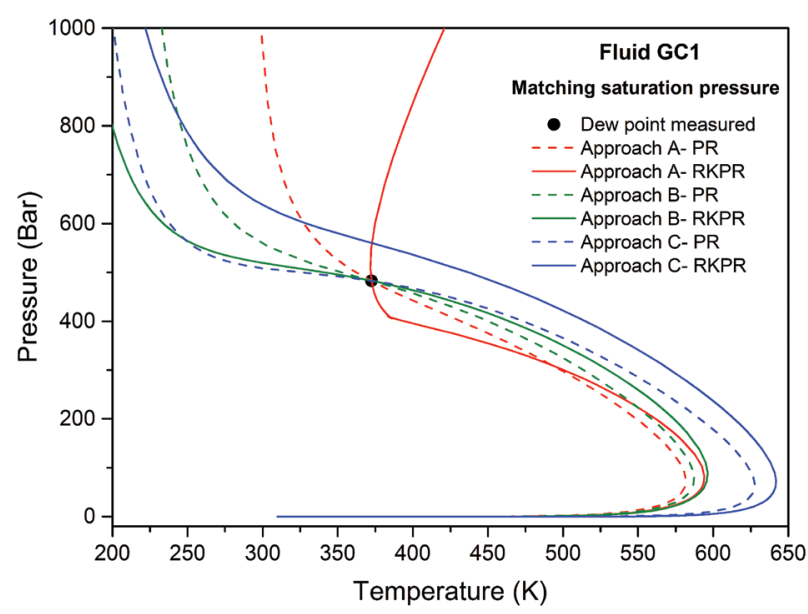

Figure 4: Predictions of the phase envelope for fluid GC1 with the PR and RKPR equations of state and the three approaches considered, when the $\mathrm{f}$ value in Table 7 multiplies all interactions in order to match the experimental saturation pressure at the reservoir temperature.

Table 7: Matching of saturation pressure: Multiplying factors $f$ for interaction parameters of the PR and RKPR equations of state, with the different fluids studied in this work.

\begin{tabular}{|c|c|c|c|c|c|c|}
\hline & \multicolumn{2}{|c|}{ 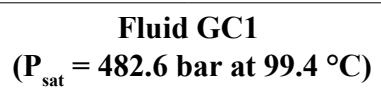 } & \multicolumn{2}{|c|}{ 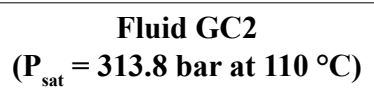 } & \multicolumn{2}{|c|}{$\begin{array}{c}\text { Fluid VO } \\
\left(\mathrm{P}_{\text {sat }}=\mathbf{2 6 8 . 0 \text { bar at } 1 0 0}{ }^{\circ} \mathrm{C}\right)\end{array}$} \\
\hline & PR & RKPR & PR & RKPR & PR & RKPR \\
\hline Approach A & 1.6707 & 1.4879 & 2.1294 & 1.8521 & 1.6461 & 1.3454 \\
\hline Approach B & 0.9635 & 0.5 & 1.5515 & 2.2340 & 0.6709 & 0.9800 \\
\hline Approach C & 0.6383 & 0 & 1.3267 & 1.4913 & 0.6715 & 0.9844 \\
\hline
\end{tabular}




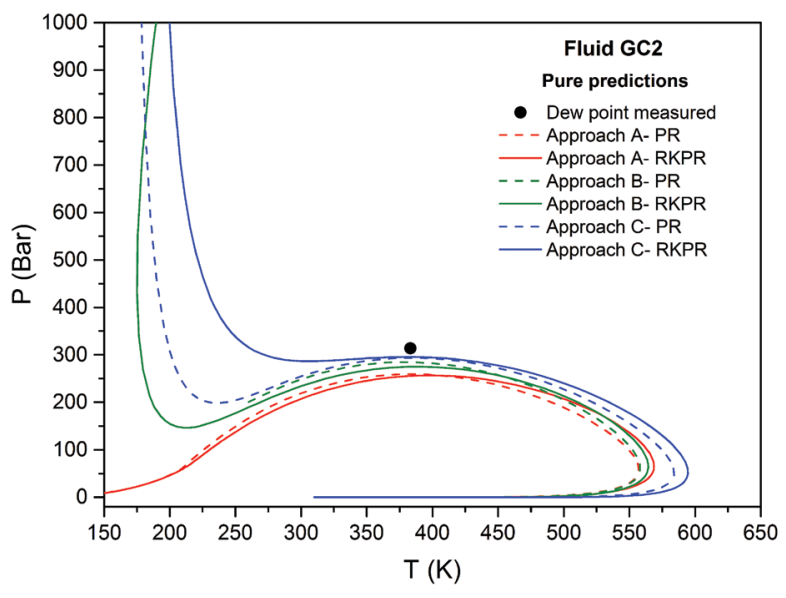

Figure 5: Pure predictions of the phase envelope for fluid GC2, with the PR and RKPR equations of state and the three approaches considered. Default interactions for alkanes assigned to all fractions.

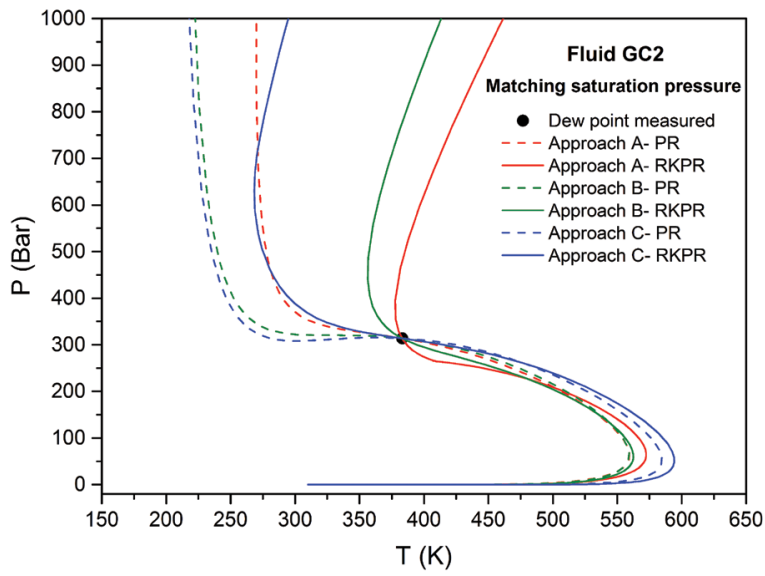

Figure 6: Predictions of the phase envelope for fluid GC2 with the PR and RKPR equations of state and the three approaches considered, when the $\mathrm{f}$ value in Table 7 multiplies all interactions in order to match the experimental saturation pressure at the reservoir temperature.

Accordingly, the f values in Table 7 show the opposite evolution: Approach A always requires the highest $\mathrm{f}$ values in order to match the experimental pressure, while Approach $\mathrm{C}$ always requires values lower than one, given the overestimation obtained from pure predictions. Two partial exceptions can be observed: The first one involves a very high $\mathrm{f}$ value for the GC2 fluid with RKPR and approach B, denoting a low sensitivity of the envelope around the reservoir temperature, probably related to the combination of kij and lij interactions. The second appears in the volatile oil (VO), where there is practically no change in the predicted

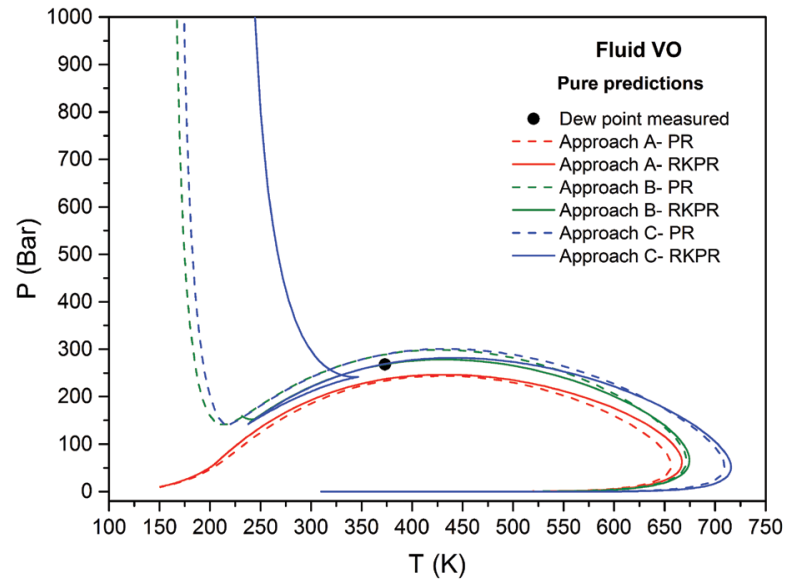

Figure 7: Pure predictions of the phase envelope for fluid VO, with the PR and RKPR equations of state and the three approaches considered. Default interactions for alkanes assigned to all fractions.

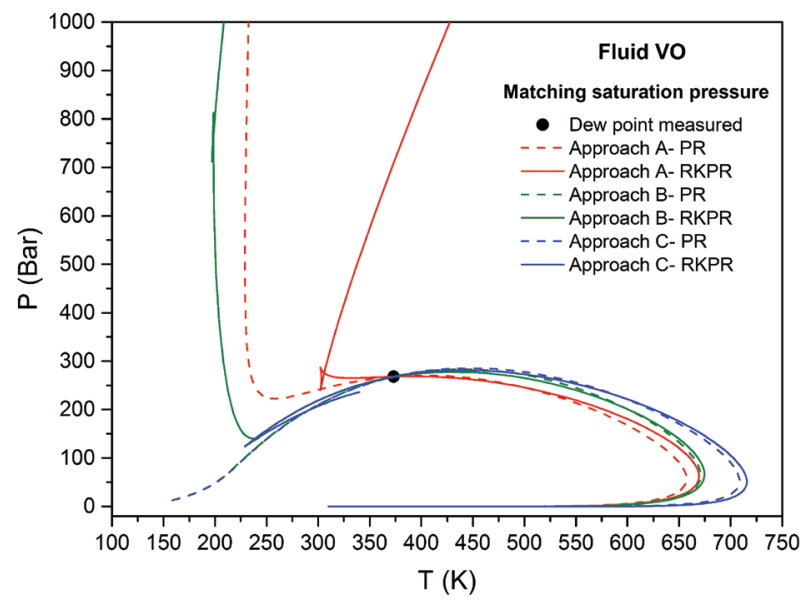

Figure 8: Predictions of the phase envelope for fluid VO with the PR and RKPR equations of state and the three approaches considered, when the $\mathrm{f}$ value in Table 7 multiplies all interactions in order to match the experimental saturation pressure at the reservoir temperature.

saturation pressure when going from approach B to C, i.e., there is practically no effect from decomposing the $\mathrm{C} 20+$ fraction in this regard.

It is important to remark that predictions corresponding to Approaches B and C in Figs. 3, 5 and 7 should not be considered as a demonstration of the predictive potential of these approaches, since such potential could only be developed when appropriate interaction parameters are defined considering the particularities of each fluid, that can be more aromatic or paraffinic, for example through correlations with molecular 
weight and density of each fraction. Nevertheless, approaches $\mathrm{B}$ and $\mathrm{C}$ have shown a reasonable predictive capacity for the saturation pressures available, especially with RKPR for the volatile oil.

Once again, we emphasize that the three approaches implemented in this work were defined in order to study the effect of three different factors separately: representation of the residual fraction, pure (pseudo-) component parameters and interaction parameters. Indeed, results in Figs. 3 to 8 show that all of them play an important role in the fluids investigated, and that the effects are even more appreciable for the gas condensates than for the volatile oil. Moreover, the same types of effects are observed either with the PR or the RKPR EoS.

A specific observation regarding the limitations of Approach A can be made based on the results in Fig. 8. While the upper part of a gas condensate phase envelope can go either up or down in pressure as temperature decreases, depending on the case (the divergence to higher pressures occurring for the most asymmetric mixtures that show liquid-liquid like immiscibility), it most frequently goes down for a volatile or (especially) black oil. This expected behaviour is indeed the one observed in Fig. 8 with approaches B and C, when the saturation pressure has been matched through adjustment of the interaction coefficients (the only exception is given by approach B with RKPR, but liquid-liquid separation develops only up to temperatures around $200 \mathrm{~K}$ ). Nevertheless, the same procedure leads to the artificial prediction of what could be a wrong behaviour with approach A, apparently forced by the large values of interaction parameters that were required to match the experimental saturation point. It is interesting to see that this happens with both models, even when they were parameterized very differently: only constant kij values for the PR EoS (the classic way) and lij plus some temperature dependent kij for the RKPR EoS (see Cismondi Duarte et al., 2015). It should be noted that the crossings appearing in some of the envelopes calculated with the RKPR EoS for the VO fluid denote three-phase behaviour, always below the reservoir temperature, but we do not have evidence to confirm or discard this possibility.

Other strong reasons for which it could be an important mistake to represent the different SCN fractions as n-alkanes are exposed in Fig. 9, which shows how the $\mathrm{M}-\rho$ coordinates of real reservoir fluids are located at important distances from the n-alkanes curve.

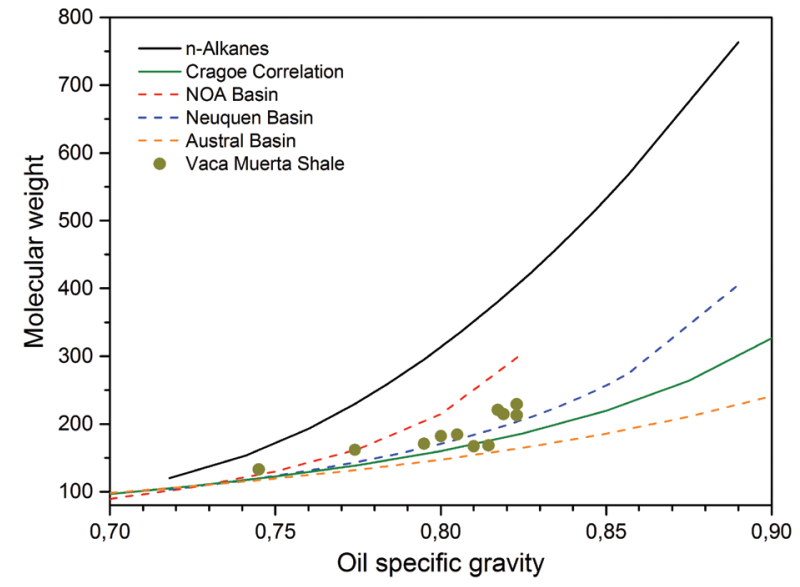

Figure 9: Data for reservoir fluids and their fractions, from three of the main basins in Argentina (Canel and Mediavilla, 1990), in comparison to the n-alkanes molecular weight - density characteristic curve and the Cragoe Correlation (Craft and Hawkins, 1991). Points correspond to wells from the Vaca Muerta formation.

Figures 10 and 11 show the predicted liquid drop-out curves for the $\mathrm{GC} 1$ and $\mathrm{GC} 2$ fluids, while the corresponding pressure-volume curves are presented in Figs. 12 and 13. In all cases volume shift correlations were implemented. Predicted curves are shown in comparison to experimental data obtained from the CCE tests, as described before in the experimental section. Note that even when the reproduction of the saturation pressure is imposed on all approaches and models, the predicted retrograde behaviour below such common point can be very different from one case to another (Figs. 10 and 11). The same observations regarding the influence of all factors studied apply here, as for the prediction of phase envelopes. On the contrary, all pressure-relative volume curves look very similar in Figs. 12 and 13. In the case of fluid GC2 (Fig. 13) curves seem to be affected only by the EoS and not by the approach, with a better approximation to the experimental curve by the RKPR EoS.

On the other hand, something that is specific to the retrograde condensation curves and could not be directly appreciated in the effects on phase envelopes (even less in the volumetric behaviour), is the difference in slopes between approaches $\mathrm{B}$ and $\mathrm{C}$ when the condensation starts at the saturation point. As could be expected, the slopes obtained with Approach $\mathrm{C}$ are less pronounced (and more in accordance with the experimental shape of the curve) than the ones corresponding 
to Approach B with the same model. The effect is perhaps clearer for $\mathrm{GC} 2$, with the $\mathrm{B}-\mathrm{C}$ crossings at lower pressures, and also higher maximum values of condensation with Approach $\mathrm{C}$, for which results are closer to the experimental. The same is valid for GC1 with PR and, in a way, also with RKPR except for the shift in the curve for approach $\mathrm{C}$, due to not matching the saturation pressure.

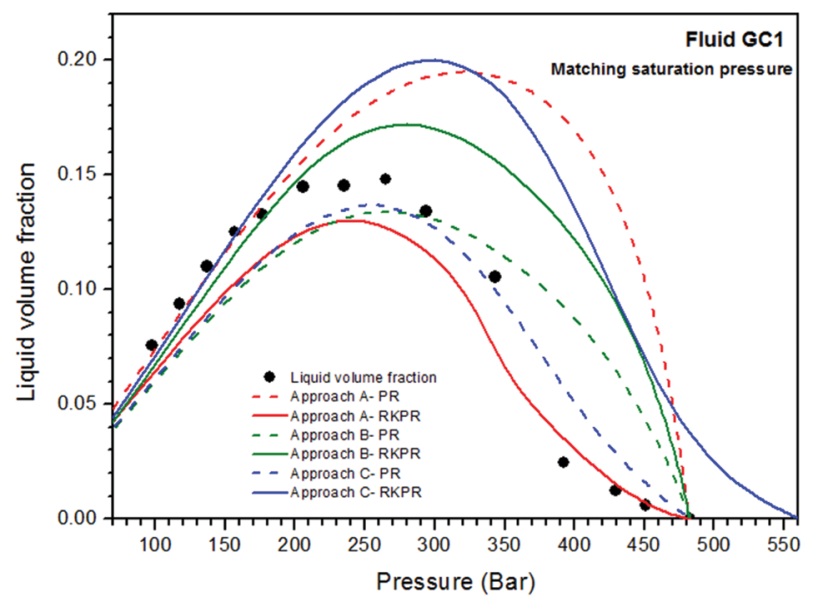

Figure 10: Predictions of the liquid drop-out curve for fluid GC1 at 372.55 $\mathrm{K}$, with the PR and RKPR equations of state and the three approaches considered. The $f$ value in Table 7 multiplies all interactions in order to match the experimental saturation pressure. Black dots represent measurements.

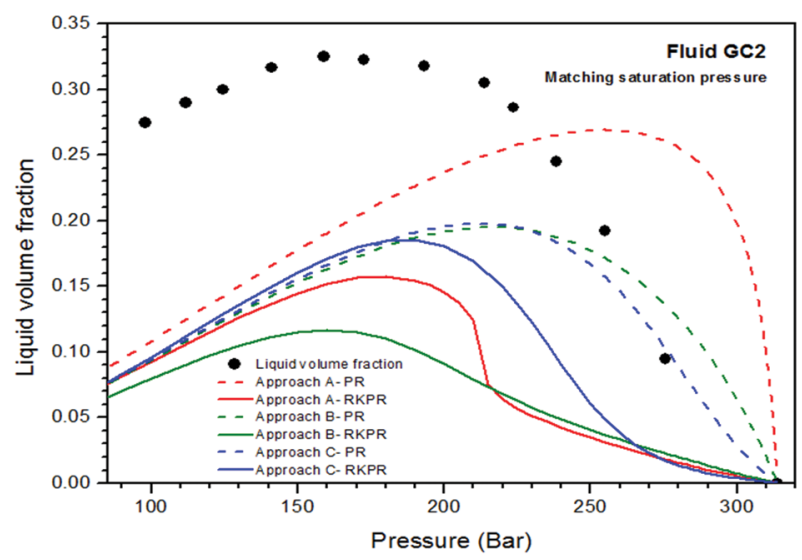

Figure 11: Predictions of the liquid drop-out curve for fluid GC2 at 383.15 $\mathrm{K}$, with the PR and RKPR equations of state and the three approaches considered. The $f$ value in Table 7 multiplies all interactions in order to match the experimental saturation pressure. Black dots represent measurements.

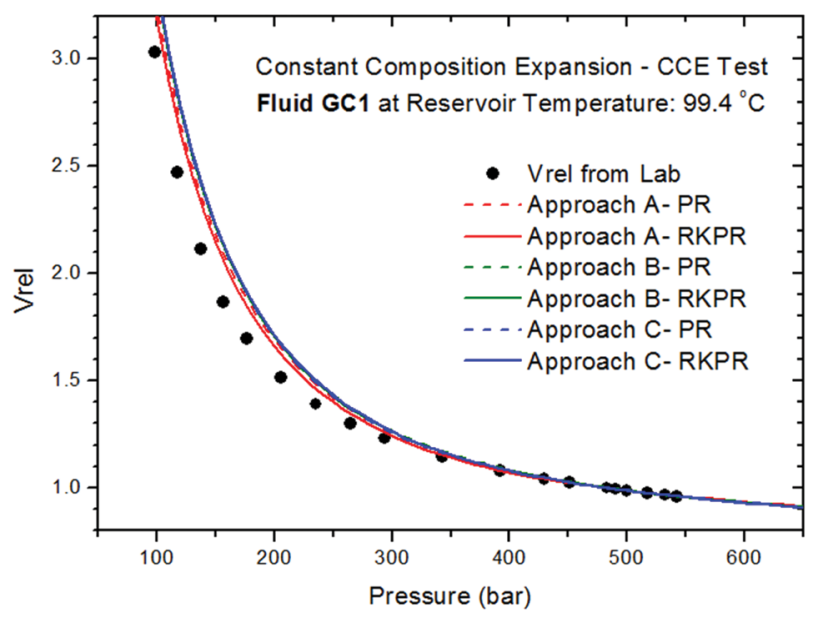

Figure 12: Predictions for the CCE Pressure-Volume curve for fluid GC1 at $372.55 \mathrm{~K}$, with the PR and RKPR equations of state and the three approaches considered. The $f$ value in Table 7 multiplies all interactions in order to match the experimental saturation pressure. Black dots represent measurements

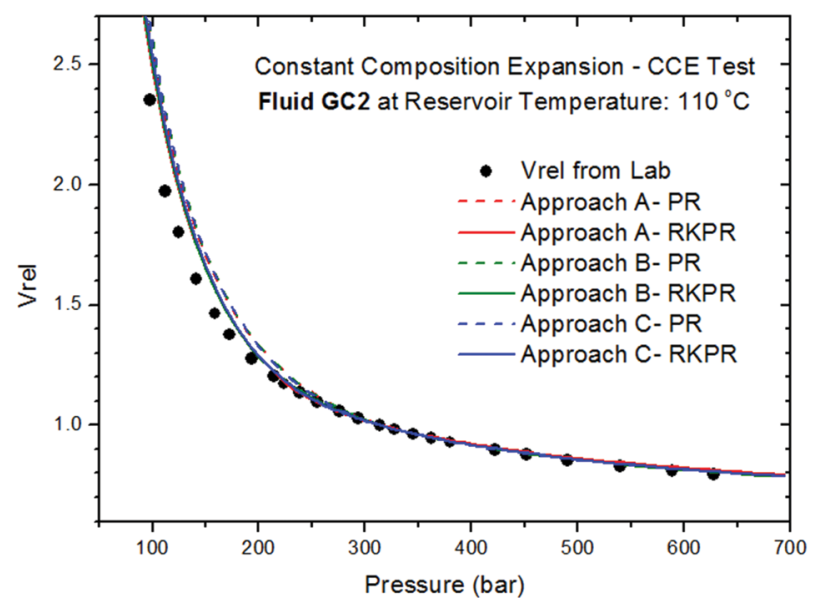

Figure 13: Predictions for the CCE Pressure-Volume curve for fluid GC2 at $383.15 \mathrm{~K}$, with the PR and RKPR equations of state and the three approaches considered. The $f$ value in Table 7 multiplies all interactions in order to match the experimental saturation pressure. Black dots represent measurements.

This improvement would confirm the effect and necessity of decomposing the $\mathrm{C} 20+$ fraction.

What was not expected are the correct shapes of the curves predicted with Approach A (only with the RKPR EoS), even with a reasonable quantitative agreement with the data for the GC1 fluid in Fig. 10. So far, we do not have an explanation for this. 


\section{CONCLUSIONS}

Compositional and PVT data have been reported for two gas condensate fluids from the Vaca Muerta formation in Argentina. Motivated by the fact that each composition was analyzed only by chromatography, without making density and molecular weight measurements for each single fraction, a new methodology was developed to estimate these properties from C6 to $\mathrm{C} 20+$, based on selected functionalities with carbon number and on measured values for the fluid. The proposed strategy avoids use of the unique generalized values from Katz and Firoozabadi (1978) and would allow, instead, capturing the different characteristics of each fluid. It was applied to the two gas condensates, and also a third fluid classified as volatile oil.

A thermodynamic modelling study was carried out for the three fluids, based on the PR and RKPR equations of state and three different approaches designed to evaluate the effects of different factors or degrees of freedom involved in this type of modelling. In approach A each SCN fraction is treated as a normal alkane, while correlations based on density and molecular weight are used to estimate each fraction parameters in approaches B an C. Only in approach C a split of the $\mathrm{C} 20+$ fraction into five pseudo-components is performed.

Clear differences were found from the application of approaches A, B and C, and different observations were made that could help to define a more specific and complete methodology aiming at the quantitative description of the PVT behaviour of this type of fluids. Both the phase envelopes and isothermal retrograde condensation curves turned out to be quite sensitive to the modelling approach, but not the CCE pressure-relative volume curves. In particular, the best qualitative representation of the experimental retrograde behavior in the CCE experiment for the gas condensates was obtained with approach $\mathrm{C}$. This involves especially the higher pressure region, where the heaviest fractions condense first, and would be a consequence of the more detailed treatment of the $\mathrm{C} 20+$ fraction through 5 different pseudo-components. Much better quantitative results are expected for the near future if some RKPR parameters are correlated with properties of the different fluid cuts, like density and molecular weight.

\section{ACKNOWLEDGEMENTS}

We want to thank Osvaldo Migliavacca for his work in the PVT lab.

We also acknowledge the financial support received from the following Argentinean institutions: Consejo
Nacional de Investigaciones Científicas y Técnicas de la República Argentina (CONICET), Agencia Nacional de Promoción Científica y Tecnológica (ANPCyT), and Universidad Nacional de Córdoba (UNC).

\section{NOMENCLATURE}

\author{
Abbreviations \\ CCE: Constant Composition Expansion. \\ $C M E$ : Constant Mass Experiment. \\ $C N$ : Carbon Number. \\ EoS: $\quad$ Equation of State \\ FDC: $\quad$ Field Development Consultants (FDC de \\ Argentina SRL) \\ GCl: $\quad$ Gas Condensate fluid $\left(\mathrm{N}^{\circ} 1\right)$. \\ GC2: $\quad$ Gas Condensate fluid $\left(\mathrm{N}^{\circ} 2\right)$. \\ GOR: Gas-Oil Ratio. \\ $H P$ : Hewlett-Packard \\ ITBA: Instituto Tecnológico de Buenos Aires \\ $P R$ : $\quad$ Peng-Robinson equation of state. \\ $P V: \quad$ Pressure-Volume. \\ PVT: $\quad$ Pressure, Volume and Temperature. \\ RKPR: Generalized Redlich-Kwong-Peng- \\ Robinson equation of state. \\ $S C N$ : Single Carbon Number. \\ TBP: $\quad$ True Boiling Point \\ $V O: \quad$ Volatile Oil fluid.
}

Roman letters

A: $\quad$ Constant in Eq. (4) for the estimation of a SCN mole fraction.

$A_{d}$ : $\quad$ Constant in Eq. (2) for the estimation of a SCN fraction density.

$B$ : $\quad$ Constant in Eq. (4) for the estimation of a SCN mole fraction.

$B_{d}$ : $\quad$ Constant in Eq. (2) for the estimation of a SCN fraction density.

$C$ : $\quad$ Constant in Eq. (1) for the estimation of a SCN fraction molecular weight.

$\mathrm{Ci}$ ( $i=6$ to 19): Hydrocarbon cut or fraction of the reservoir fluid.

$\mathrm{Ci}+(i=6,7,10,20,30$ or 35): Residual fraction of the reservoir fluid, containing the SCN fractions starting from $i$ and higher.

Cmax: For a given distribution of the $\mathrm{C} 20+$ fraction, the maximum carbon number where the summation reaches $z_{20+}$. 
$f: \quad$ factor involved in the matching mode.

$i$ : number identifying a hydrocarbon SCN cut or fraction.

$k_{i j}: \quad$ attractive interaction parameter between compound "i" and compound " $\mathrm{j}$ ".

$l_{i j}: \quad$ repulsive interaction parameter between compound "i" and compound " $\mathrm{j}$ ".

M: $\quad$ Molecular weight.

$m$ : $\quad$ Collected mass.

$M_{20+}$ : $\quad$ Molecular weight of the residual fraction $\mathrm{C} 20+$.

Nps: $\quad$ Number of pseudo-components.

$P: \quad$ Pressure.

T: $\quad$ Temperature.

$V$ : Volume.

$V_{6+}: \quad$ The volume occupied by the C6+ fraction per mole of total fluid. Since it is defined just as an auxiliary variable in the procedure of assigning density values for the different $\mathrm{SCN}$ and residual fractions, it refers to the same standard conditions for which densities are defined.

$z: \quad$ Mole fraction.

$z_{6+}: \quad$ Mole fraction of the residual fraction C6+.

$z_{20+}: \quad$ Mole fraction of the residual fraction $\mathrm{C} 20+$.

Greek letters

$\rho \quad$ Density.

$\rho_{20+} \quad$ Density of the residual fraction C20+.

Super/subscripts

c Critical property.

calc Calculated property.

$i$ number identifying a hydrocarbon SCN cut or fraction.

sat saturation state.

\section{REFERENCES}

Beckwith, R., Shale Gas: Promising Prospects Worldwide. Journal of Petroleum Technology, 63(07), 37-40 (2011).
Beckwith, R., The trend toward shale oil. Journal of Petroleum Technology, 63(7), 42-43 (2011).

Canel C. and Mediavilla J., Estudios PVT a partir de datos de boca de pozo. Proceedings of the 2das Jornadas de Informática Aplicada a la Producción. Argentina (1990).

Cismondi, M. and Mollerup, J., Development and Application of a Three-Parameter RK-PR Equation of State. Fluid Phase Equilibria, 232, 74-89 (2005).

Cismondi Duarte, M., Cruz Doblas, J., Gomez, M.J., Montoya, G.F., Modelling the phase behavior of alkane mixtures in wide ranges of conditions: New parameterization and predictive correlations of binary interactions for the RKPR EOS. Fluid Phase Equilibria, 403, 49-59 (2015).

Craft, B. C., and Hawkins, M., Applied Petroleum Reservoir Engineering, Prentice Hall PTR, Englewood Cliffs, NJ 07632, $2^{\text {nd }}$ ed. (1991).

Danesh, Ali, PVT and Phase Behavior of Petroleum Reservoir Fluids, Elsevier (1998).

Jaubert, J-N, Avaullee, L. and Souvay, J-F. A crude oil data bank containing more than 5000 PVT and gas injection data. Journal of Petroleum Science and Engineering 34, 65-107 (2002).

Katz, D.L. and Firoozabadi, A., Predicting phase behavior of condensate/crude-oil systems using methane interaction coefficients, J. Petroleum Technol. 20, 1649-1655 (1978).

Pedersen, K.S., Blilie, A.L., Meisingset, K.K., PVT calculations on petroleum reservoir fluids using measured and estimated compositional data for the plus fraction, Ind. Eng. Chem. Res. 31, 1378-1384 (1992).

Pedersen, K.S. and Christensen, P.L. , Phase Behavior of Petroleum Reservoir Fluids, CRC/Taylor \& Francis, Boca Raton (2006).

Peng, D.-Y., Robinson, D.B., A New Two-Constant Equation of State, Industrial \& Engineering Chemistry Fundamentals, 15, 59-64 (1976).

Ramello, J.I. and Cismondi, M., On the estimation of carbon number distributions in reservoir fluids heavy fractions: Revision of the logarithmic relation and proposition of a new simple and effective approach.. Unpublished work (2016) 
